
\title{
BReserach S Sulue \\ Flavonoids from bamboo drive seasonal changes to the gut microbiome of giant pandas
}

\section{Le Wang}

Guangping Huang

Rong Hou

Dunwu Qi

Qi Wu

Yonggang Nie

Zhenqiang Zuo

Rui Ma

Wenliang Zhou

Yingjie Ma

Yibo Hu

Zhisong Yang

Li Yan

Fu-Wen Wei

\section{Video Byte}

Keywords: Microbiome, plant secondary metabolites, PSMs, flavonoids, giant pandas, metabolomics, metagenomics, gut microbiome, absorption, metabolism, diet, conservation

Posted Date: October 13th, 2021

DOI: https://doi.org/10.21203/rs.3.rs-967536/v1

License: (9) This work is licensed under a Creative Commons Attribution 4.0 International License. Read Full License 


\section{Abstract}

Flavonoids are ubiquitous, nutritionally non-essential natural products of plants and they are frequently used to promote health. However, flavonoid metabolism and the impact of flavonoids on gut microbiomes are not well understood. Bamboo is particularly flavonoid rich, making giant pandas, with their all-bamboo diet, a unique research target. A recent study examined the diet, feces, and plasma of giant pandas to get a comprehensive view of flavonoid metabolism and its impact on the gut microbiome. The researchers found that bamboo leaves had more flavonoids than bamboo shoots. They also found that only a small fraction of dietary flavonoids were absorbed into the bloodstream and that the gut microbiota extensively utilized and transformed the flavonoids. The seasonal shifts in the flavonoid profile of bamboo drove changes to the gut microbial composition. Specifically, the gut microbial diversity and the abundance of virulence factors were lower during seasons with higher flavonoid content and the abundance of cellulose-degrading bacterial species was higher in those seasons. These results revealed novel interactions between flavonoids and the gut microbiome in pandas and suggest that flavonoids should be explored as a prebiotic in conservation management. 